\title{
Capillary electrochromatographic separation of non-steroidal anti-inflammatory drugs with a histidine bonded phase
}

\author{
Yi-Fen Pai, Chuen-Ying Liu* \\ Department of Chemistry, National Taiwan University, Taipei, Taiwan
}

Received 6 May 2002; received in revised form 20 September 2002; accepted 23 September 2002

\begin{abstract}
An open tubular wall-coated capillary column containing histidine functional groups was prepared and employed for the capillary electrochromatographic separation of non-steroidal anti-inflammatory drugs. The anion exchange along with the hydrogen bonding and hydrophobic properties of the surface coating allowed the separation of analytes with very similar ionic mobility. Selectivity and resolution were studied by changing the $\mathrm{pH}$ over the range from 3.5 to 5.0 and the concentration of the buffer from 10 to $25 \mathrm{mM}$, as well as variation of the organic modifier, such as methanol, ethanol and 1-propanol over the range 7.5 to $20 \%$. The optimum experimental conditions for the separation of a drug mixture, which consisted of indoprofen, ketoprofen, suprofen, naproxen, flurbiprofen, fenoprofen and ibuprofen were using a mixture of acetate buffer $(20 \mathrm{mM}, \mathrm{pH} 5.0)$-ethanol $(1: 5, \mathrm{v} / \mathrm{v})$ as background electrolyte and an applied voltage of $-20 \mathrm{kV}$ with UV detection at $220 \mathrm{~nm}$. The separation of these drugs could be achieved with an average plate number of $1.0 \cdot 10^{5} \mathrm{~m}^{-1}$.

(c) 2002 Elsevier Science B.V. All rights reserved.
\end{abstract}

Keywords: Electrochromatography; Capillary columns; Stationary phase, electrochromatography; Non-steroidal antiinflammatory drugs

\section{Introduction}

In the case of long-term non-steroidal anti-inflammatory drug (NSAID) therapy, a high incidence of severe side effects, including gastrointestinal ulcers and nephrosis, has been reported, especially in rheumatoid arthritis (RA) therapy. It is also of concern that many medications may interact with other drugs when they are frequently co-administered to RA patients. Hence it is considered that the monitoring of NSAIDs is of importance in order to

\footnotetext{
*Corresponding author. Tel.: +886-22-363-0231; fax: +88622-363-8543.

E-mail address: cyliu@ccms.ntu.edu.tw (C.-Y. Liu).
}

improve the toxicological management of long term NSAID therapy [1]. In other words, for clinical studies and drug quality control purposes, it is essential to establish accurate, sensitive and selective analytical techniques that permit detection and quantitative measurement of drug entities in biological and pharmaceutical samples [2].

A number of high-performance liquid chromatography (HPLC) methods for the determination of NSAIDs in biological samples have appeared in the literature, such as those involving UV detection [1,35], photodiode-array detection [6], electrochemical detection [7] or liquid chromatography-mass spectrometry (LC-MS) assay [2]. Capillary electrophoresis (CE) has been increasingly used for separation 
because of its high separation efficiency in comparison with HPLC. Separations of NSAIDs by CE optimized by half-fraction factorial designs [8], using non-aqueous systems [9-11], by micellar electrokinetic capillary chromatography (MEKC) [12-14] and automatic on-line integration of solid-phase extraction and CE [15] have been described. Cyclodextrin and its derivatives as chiral additives for the CE separation of NSAIDs have also been reported [10,16-18].

Capillary electrochromatography (CEC) has been drawing increasing attention recently. CEC offers wider selectivity and enables separation of neutral compounds as well as charged compounds. A capillary packed with RP-18 silica particles was reported for the separation of NSAIDs [19]. Hoegger and Freitag synthesized an acrylamide-based monoliths as robust stationary phases for the separation of NSAIDs [20]. Liu et al. recently reported a phenomenological approach to describe the retention behaviors of solutes in CEC [21]. Some of the model compounds were NSAIDs and the CEC systems studied included both open tubular and packed columns.

Histidine has net charge of -1 at $\mathrm{pH}$ above 10 , the only charged group is the carboxylate anion. At $\mathrm{pH}$ around 5, histidine has a net charge of +1 because the imidazole group is protonated. At very low $\mathrm{pH}$ values, the histidine molecule has a net positive charge of 2 , while at its isoelectric point $(\mathrm{p} I=7.6)$, the molecule carries no net charge. In addition to the properties of hydrophobic force, hydrogen bonding and anion exchange which can be made use of to separate the analytes, the above mentioned property could form the basis of an electrophoretic separation. Histidine-functionalized silica and its copper complex have been investigated as the stationary phases of packed CEC for the separation of both organic and inorganic anions [22]. Chemically bonding histidine groups to the fusedsilica capillary wall for the separation of plant phenolics has also been studied [23]. A major challenge in CEC is the understanding of the interaction mechanism and hence the choice of a suitable stationary phase for a given analyte mixture. In this work, we would like to report our recent investigation of the chromatographic behavior of histidine-coated capillary column for the separation of NSAIDs.

\section{Experimental}

\subsection{Apparatus}

All experiments were carried out using a laboratory-built unit. It consists of a $\pm 30 \mathrm{kV}$ high-voltage power supply (Gamma High Voltage Research, Ormond Beach, FL, USA), a UV-visible detector (Model L-4200, Hitachi, Japan) and an integrator (Model D-2500, Hitachi). Fused-silica capillaries (75 $\mu \mathrm{m}$ I.D., Polymicro Technologies, Phoenix, AZ, USA) were typically flushed with $1 M \mathrm{NaOH}$ for 30 $\min$.

\subsection{Reagents and chemicals}

Most chemicals were of analytical-reagent grade from Merck (Darmstadt, Germany). Purified water $(18 \mathrm{M} \Omega \mathrm{cm})$ from a Milli-Q water purification system (Millipore, Bedford, MA, USA) was used to prepare all solutions. Indoprofen, ketoprofen, naproxen, ibuprofen, fenoprofen, flurbiprofen, suprofen and histidine were obtained from Sigma (St. Louis, MO, USA). Acetic acid, hydrochloric acid (Merck), sodium acetate and sodium hydroxide (Wako, Japan), benzyl alcohol, acetone and methanol (Acros, Geel, Belgium), 3-chloropropyltrimethoxysilane (Aldrich, Milwaukee, WI, USA) and $m$-xylene (Janssen, Belgium) were purchased from the indicated sources.

Stock solutions $(0.01 M)$ of the NSAIDs were prepared in methanol and diluted appropriately with methanol prior to use. All solvents and solutions for CEC analysis were filtered through a $0.45 \mu \mathrm{m}$ PTFE (Millipore) or cellulose acetate membrane (Whatman).

\subsection{Column preparation}

The detailed procedures for the preparation of the wall-coated histidine capillary column has been described previously [23]. Fused-silica capillaries (75 $\mu \mathrm{m}$ I.D.) were first flushed with $1 \mathrm{M} \mathrm{NaOH}(30$ $\mathrm{min})$, then pure water $(15 \mathrm{~min}), 1 \mathrm{M} \mathrm{HCl}(30 \mathrm{~min})$ and pure water $(15 \mathrm{~min})$. Before silanization the capillaries were rinsed with methanol $(5 \mathrm{~min})$ and then dried in a gas chromatography oven at $110^{\circ} \mathrm{C}$ for $1 \mathrm{~h}$ under a nitrogen flow of $2.5 \mathrm{~kg} \mathrm{~cm}^{-2}$. The silanol group of the capillary column was activated with 3 -chlorotrimethoxysilane in $m$-xylene $(10 \%$, v/ 
v) at $110^{\circ} \mathrm{C}$, then functionalized with histidine in the GC oven at $150{ }^{\circ} \mathrm{C}$. The resulting column was flushed successively with acetone and pure water at ambient temperature.

\subsection{Electrophoresis conditions}

Before analysis, the coated capillaries were preconditioned with the running buffer. They were rinsed with methanol, pure water and buffer between runs at 1 or $2 \mathrm{~min}$ intervals. The samples were injected by siphoning at a height difference of $10 \mathrm{~cm}$ for $10 \mathrm{~s}$. The electroosmotic flow (EOF) was measured with benzyl alcohol. The samples were detected at $220 \mathrm{~nm}$.

\section{Results and discussion}

\subsection{Effect of $p H$ on the separation}

The common structural feature of NSAIDs is an arylpropionate moiety (Fig. 1). The $\mathrm{p} K_{\mathrm{a}}$ values of most analytes are around 4 . The bonded groups on the capillary wall were derived from an amphoteric substance, histidine (p $\left.K_{\mathrm{a}}: 1.78,5.97,8.97\right)$ (Fig. 2). Hence the $\mathrm{pH}$ of the background electrolyte (BGE)

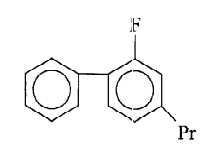

Flurbiprofen (MW 244.3; pKa 4.13)

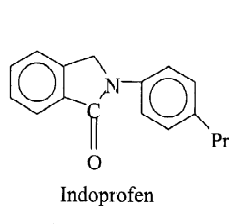

$(281.3 ; 4.29)$

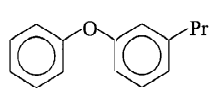

Fenoprofen

(242.2)

Fig. 1. Structures of non-steroidal anti-inflammatory drugs (NSAIDs).

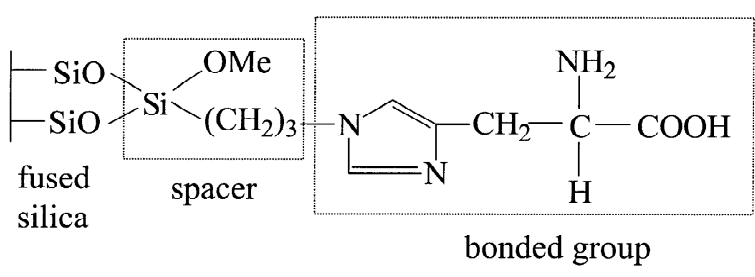

Fig. 2. Suggested structure of the histidine bonded phase.

governs not only the charge of the analyte, but also the charge of the wall coated histidine groups. Under more acidic condition, a greater fraction of the NSAIDs exist as neutral species, while the histidine bonded groups become more protonated. For maximizing the electrostatic interaction with the stationary phase as well as having large variation among the mobilities of the analytes, a weak acidic medium seems to be useful for the separation. In the preliminary study, acetate buffer-methanol mixture was chosen as the BGE. At $\mathrm{pH}$ values over the range of 3.5 to 4.5 , there was no resolution of the pairs of indoprofen-ketoprofen and flurbiprofen-suprofen. However, increasing the $\mathrm{pH}$ to 5.0 dramatically improved the resolution of these pairs.

In this work, EOF reversal was an attribute to the positive charge of the histidine groups on the capillary surface at a $\mathrm{pH}$ below its isoelectric point. The EOF mobility was slightly reduced with increasing $\mathrm{pH}$. Values were $1.94 \cdot 10^{-4}, 1.50 \cdot 10^{-4}, 1.31 \cdot 10^{-4}$ and $1.09 \cdot 10^{-4} \mathrm{~cm}^{2} \mathrm{~V}^{-1} \mathrm{~s}^{-1}$ at $\mathrm{pH} 3.5,4.0,4.5$ and 5.0 , respectively. Here, the solutes were separated using a negative voltage. Therefore, a slower migration with increase of $\mathrm{pH}$ was demonstrated. Fig. 3 shows how variation in $\mathrm{pH}$ from 3.5 to 5.0 can optimize the separation of seven analytes. At $\mathrm{pH}$ below 4.5, some of the analytes have similar electrophoretic mobility and are therefore not well resolved. At $\mathrm{pH}$ around 5, the electrophoretic mobilities are far enough apart so that these drugs can be well separated.

\subsection{Effect of concentration}

In order to investigate the effect of the electrolyte ionic strength, the concentration of acetate buffer was varied over the range of $10-25 \mathrm{mM}$, while keeping the methanol percentage at $15 \%$. The EOF mobility only slightly decreased with increasing buffer concentration, $1.40 \cdot 10^{-4}, 1.25 \cdot 10^{-4}, 1.09$. 


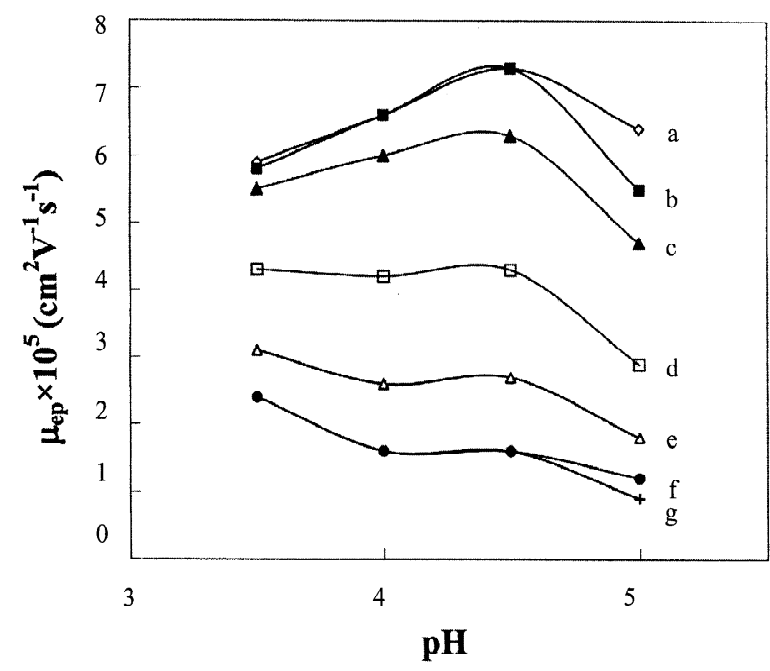

Fig. 3. Dependence of electrophoretic mobility on the buffer $\mathrm{pH}$. Column: histidine bonded phase capillary, $90 \mathrm{~cm}(70 \mathrm{~cm}$ to detector $) \times 75 \mu \mathrm{m}$ I.D.; background electrolyte: acetate buffer $(20$ $\mathrm{m} M)$-methanol $(85: 15, \mathrm{v} / \mathrm{v})$; sample concentration: $0.5 \mathrm{~m} M$ each, except naproxen $0.25 \mathrm{mM}$; applied voltage: $-20 \mathrm{kV}$; detection: $\mathrm{UV}$ at $220 \mathrm{~nm}$. Curves: $\mathrm{a}=$ indoprofen, $\mathrm{b}=$ ketoprofen, $\mathrm{c}=$ naproxen, $\mathrm{d}=$ ibuprofen, $\mathrm{e}=$ fenoprofen, $\mathrm{f}=$ flurbiprofen, $\mathrm{g}=$ suprofen.

$10^{-4}$ and $9.5 \cdot 10^{-5} \mathrm{~cm}^{2} \mathrm{~V}^{-1} \mathrm{~s}^{-1}$, at BGE concentrations of $10,15,20$ and $25 \mathrm{~m} M$, respectively. Optimal separation was achieved with an acetate buffer of $20 \mathrm{~m} M$ (Fig. 4). A further increase in the BGE concentration has little effect on resolution. But a band broadening caused by excessive Joule heat was observed.

\subsection{Effect of selectivity on the addition of organic modifier}

NSAIDs are lipophillic acid compounds, with similar charge-to-mass ratios, a systematic investigation of the potential of nonaqueous capillary electrophoresis for the separation of NSAIDs has been reported by Cherkaoui and Veuthey [11]. The organic modifier affects dissociation constant, separation efficiency and effective electrophoretic mobility of the analytes; $\mathrm{pH}$, viscosity, dielectric constant, electroosmotic flow and conductivity of the BGE [24]. In this work, methanol, ethanol and 1-propanol were tested as modifiers of the electrolyte. It was found that higher concentration of the modifier

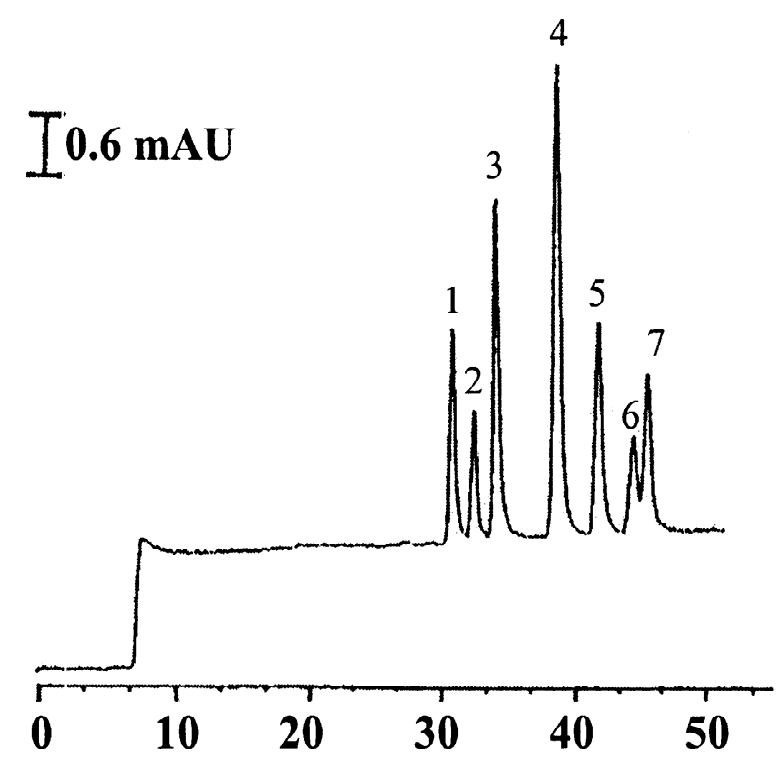

\section{Migration time (min)}

Fig. 4. Electropherogram for the separation of NSAIDs in acetate buffer ( $\mathrm{pH} 5.0,20 \mathrm{mM})$-methanol $(85: 15, \mathrm{v} / \mathrm{v})$. Conditions as in Fig. 3, except $\mathrm{pH}$ of the background electrolyte. Peak identification: $1=$ indoprofen, $2=$ ketoprofen, $3=$ naproxen, $4=$ ibuprofen, $5=$ fenoprofen, $6=$ flurbiprofen, $7=$ suprofen.

caused a decrease in the effective mobilities of all analytes (Table 1). Because the tendency of the migration of analytes to the concentration of an organic solvent is the same among three solvents employed. Therefore only the influence of the volume percentage of ethanol in the BGE on the separation of NSAIDs was shown. No data were shown for those of methanol and 1-propanol. Increasing the content of the organic modifier in the mobile phase had a positive effect on the separation (Table 1). However, it is obvious that the mixed aqueous-organic modifier buffers affect other factors including peak shape, the theoretical plate numbers and Joule heating. To obtain baseline separations for all NSAIDs, the percentage of organic modifier had to be at least $15 \%$ for methanol and $20 \%$ for ethanol, but non-baseline separation for indoprofen, ketoprofen and naproxen was observed, even at $20 \%$ of 1-propanol (Fig. 5). A poorer solubility of analytes 
Table 1

The influence of volume percentage of ethanol in background electrolyte on the separation of NSAIDs ${ }^{\mathrm{a}}$

\begin{tabular}{|c|c|c|c|c|c|c|c|c|c|c|c|c|}
\hline \multirow[t]{2}{*}{ Analyte } & \multicolumn{2}{|c|}{$7.5 \%$ (v/v) Ethanol } & \multicolumn{2}{|c|}{$10 \%(\mathrm{v} / \mathrm{v})$ Ethanol } & \multicolumn{2}{|c|}{$12.5 \%(\mathrm{v} / \mathrm{v})$ Ethanol } & \multicolumn{2}{|c|}{$15 \%(\mathrm{v} / \mathrm{v})$ Ethanol } & \multicolumn{2}{|c|}{$17.5 \%(\mathrm{v} / \mathrm{v})$ Ethanol } & \multicolumn{2}{|c|}{$20 \%$ (v/v) Ethanol } \\
\hline & $t_{\mathrm{R}}(\min )$ & $R_{\mathrm{s}}{ }^{\mathrm{b}}$ & $t_{\mathrm{R}}(\min )$ & $R_{\mathrm{s}}$ & $t_{\mathrm{R}}(\min )$ & $R_{\mathrm{s}}$ & $t_{\mathrm{R}}(\min )$ & $R_{\mathrm{s}}$ & $t_{\mathrm{R}}(\min )$ & $R_{\mathrm{s}}$ & $t_{\mathrm{R}}(\min )$ & $R_{\mathrm{s}}$ \\
\hline Indoprofen & 10.44 & - & 14.19 & - & 15.15 & - & 19.00 & - & 24.35 & - & 33.90 & - \\
\hline Ketoprofen & 10.44 & - & 14.19 & - & 15.15 & - & 19.40 & - & 25.35 & - & 35.15 & 2.50 \\
\hline Naproxen & 10.77 & - & 14.19 & - & 15.31 & - & 19.73 & - & 27.35 & 3.20 & 36.65 & 3.00 \\
\hline Ibuprofen & 11.10 & 0.85 & 14.73 & 1.08 & 15.94 & 0.90 & 20.73 & 1.60 & 29.19 & 3.68 & 39.85 & 3.88 \\
\hline Fenoprofen & 11.44 & 0.85 & 15.31 & 1.16 & 16.61 & 1.34 & 21.73 & 2.00 & 30.98 & 3.58 & 43.90 & 3.77 \\
\hline Flurbiprofen & 11.69 & 0.63 & 15.82 & 1.02 & 17.15 & 1.08 & 22.52 & 1.58 & 32.44 & 1.76 & 46.52 & 2.62 \\
\hline Suprofen & 11.69 & - & 15.82 & - & 17.15 & - & 22.70 & - & 33.44 & 0.97 & 47.73 & 1.51 \\
\hline
\end{tabular}

${ }^{a}$ Column: histidine bonded phase capillary, $90 \mathrm{~cm}(70 \mathrm{~cm}$ to detector $) \times 75 \mu \mathrm{m}$ I.D.; background electrolyte: acetate buffer $(20 \mathrm{~m} M$, pH 5.0)-ethanol; injection: hydrodynamic $(10 \mathrm{~s}, 10 \mathrm{~cm}) ;$ sample concentration: $0.5 \mathrm{mM}$ each, excep naproxen $0.25 \mathrm{~m} M$; applied voltage: $-20 \mathrm{kV}$; detection: $\mathrm{UV}$ at $220 \mathrm{~nm}$.

${ }^{\mathrm{b}} R_{\mathrm{s}}=2\left(t_{\mathrm{R} 2}-t_{\mathrm{R} 1}\right) /\left(W_{1}+W_{2}\right)$ 


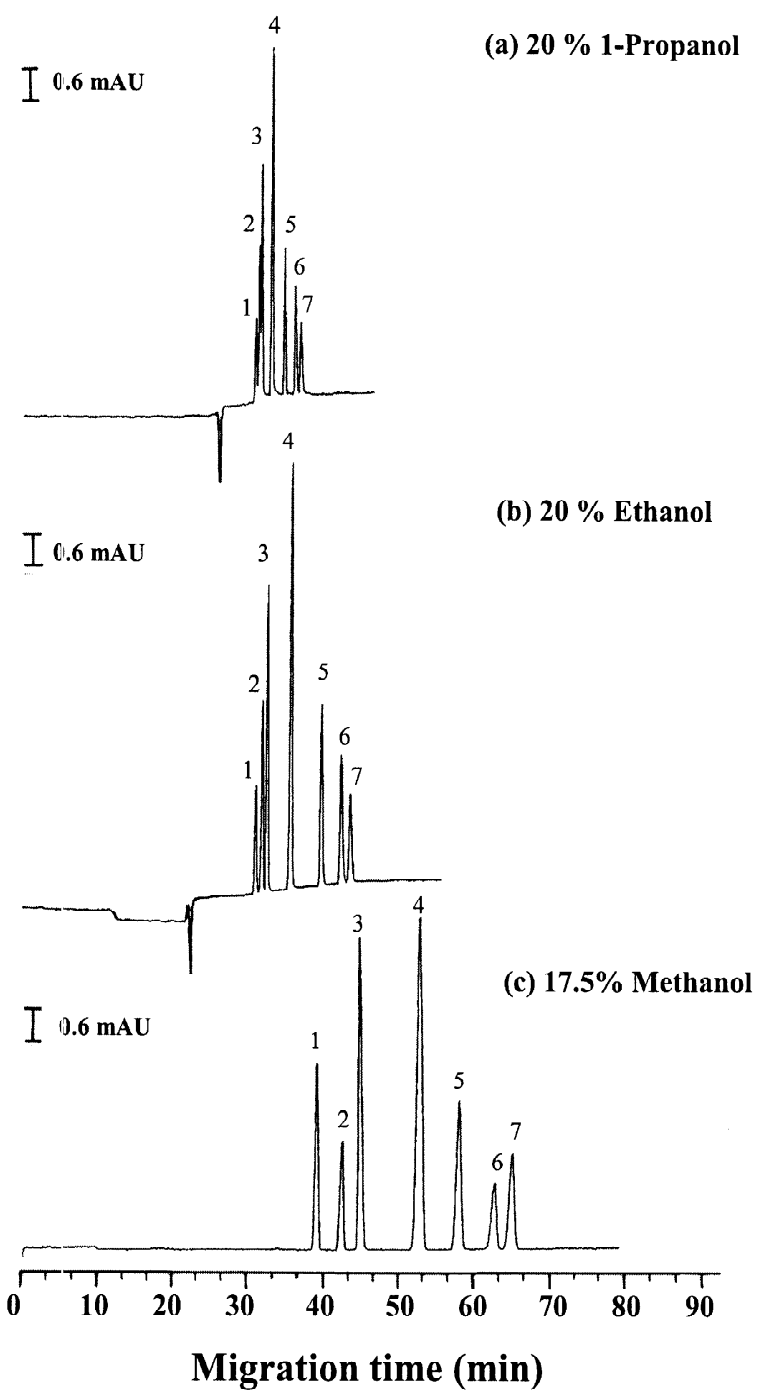

Fig. 5. Electropherograms for the separation of NSAIDs with different organic modifier at their optimal conditions. Conditions as in Fig. 4, except organic modifier in the background electrolyte. Peak identification: $1=$ indoprofen, $2=$ ketoprofen, $3=$ naproxen, $4=$ ibuprofen, $5=$ fenoprofen, $6=$ flurbiprofen, $7=$ suprofen.

in 1-propanol than in the other two solvents might be the reason.

The separation efficiencies performed with different organic modifiers at their optimal conditions are shown in Table 2. 1-Propanol gave the greatest plate number for each species, but the resolution was the poorest. In other words, from the viewpoint of the height equivalent to theoretical plate and resolution, ethanol seems the best modifier. Addition of methanol to the acetate buffer system was found to be impractical, due to excessive analysis time.

\subsection{Mechanism for the separation}

Use of the histidine bonded phase allowed a good separation of seven quite similar charge/mass ratio NSAIDs. The elution order was indoprofen $\quad$ (earliest) $>$ ketoprofen $>$ naproxen $>$ ibuprofen $>$ fenoprofen $>$ flurbiprofen $>$ suprofen. While under the same experimental conditions in untreated capillaries, no peak was found within $60 \mathrm{~min}$. If the polarity of the applied voltage was changed, namely to $+20 \mathrm{kV}$, two peaks were observed (Fig. 6). Where naproxen and flurbiprofen were first co-eluted, the other nonresolved peak contained indoprofen, ketoprofen, ibuprofen, fenoprofen, and suprofen. The different phenomena indicated that there might be some interactions of the analytes with the bonded phase.

Desiderio and Fanali [19] used a packed capillary with RP-18 silica particles for the CEC separation of NSAIDs. Under ammonium formate $(50 \mathrm{~m} M, \mathrm{pH}$ $2.5)$-acetonitrile $(40: 60, \mathrm{v} / \mathrm{v})$, the elution order was indoprofen $>$ suprofen $>$ ketoprofen $>$ naproxen $>$ fenoprofen $>$ flurbiprofen $>$ ibuprofen. In their experimental conditions, most analytes were in molecular form. The separation was based on hydrophobic discrimination. Fillet et al. [9] reported that $50 \mathrm{mM}$ ammonium acetate- $13.75 \mathrm{~m} M$ ammonia in methanol (apparent $\mathrm{pH} 8.5$ ) allows one to resolve several kinds of NSAIDs. Under this condition, most analytes are fully ionized. The elution order for the related compounds was naproxen $>$ ketoprofen $>$ flurbiprofen. With an increase in the temperature to $36^{\circ} \mathrm{C}$, the separation efficiency increased and the elution order was naproxen $>$ ketoprofen $>$ suprofen $>$ flurbiprofen $>$ indoprofen. Their work was predominantly based on electrophoretic separation.

Compared with the works mentioned above $[9,19]$, an anomalous situation is observed. This behavior might be explained through the presence of high electronegativity atoms such as fluorine, nitrogen, oxygen and sulfur on the analytes that may exhibit hydrogen bonding, dipole-dipole or Van der Waals interactions with the amino, carboxylate and imidazole groups on the bonded histidine molecules. Of 
Table 2

Separation efficiency with different organic modifiers at their optimal condition ${ }^{\text {a }}$

\begin{tabular}{|c|c|c|c|c|c|c|c|c|c|c|c|c|}
\hline \multirow[t]{2}{*}{ Analyte } & \multicolumn{4}{|c|}{ Methanol $(17.5 \%, \mathrm{v} / \mathrm{v})$} & \multicolumn{4}{|c|}{ Ethanol $(20 \%, \mathrm{v} / \mathrm{v})$} & \multicolumn{4}{|c|}{ 1-Propanol $(20 \%, \mathrm{v} / \mathrm{v})$} \\
\hline & $t_{\mathrm{R}}(\min )$ & $N^{\mathrm{b}}\left(\mathrm{m}^{-1}\right)$ & $H^{\mathrm{c}}(\mu \mathrm{m})$ & $R_{\mathrm{s}}{ }^{\mathrm{d}}$ & $t_{\mathrm{R}}(\min )$ & $N\left(\mathrm{~m}^{-1}\right)$ & $H(\mu \mathrm{m})$ & $R_{\mathrm{s}}$ & $t_{\mathrm{R}}(\mathrm{min})$ & $N\left(\mathrm{~m}^{-1}\right)$ & $H(\mu \mathrm{m})$ & $R_{\mathrm{s}}$ \\
\hline Indoprofen & 39.7 & 28000 & 32.1 & - & 33.9 & 82000 & 11.0 & - & 34.2 & 333000 & 2.7 & - \\
\hline Ketoprofen & 43.0 & 33000 & 27.3 & 3.29 & 35.1 & 88000 & 10.2 & 2.50 & 35.3 & 554000 & 1.6 & - \\
\hline Naproxen & 45.5 & 37000 & 24.3 & 2.50 & 36.6 & 96000 & 9.4 & 3.00 & 35.5 & 562000 & 1.6 & 0.38 \\
\hline Ibuprofen & 53.7 & 23000 & 39.1 & 6.56 & 39.8 & 78000 & 11.5 & 3.88 & 37.1 & 392000 & 2.3 & 1.79 \\
\hline Fenoprofen & 58.7 & 39000 & 23.0 & 3.37 & 43.9 & 113000 & 8.0 & 3.77 & 38.6 & 424000 & 2.1 & 2.96 \\
\hline Flurbiprofen & 63.4 & 46000 & 19.6 & 3.09 & 46.5 & 127000 & 7.1 & 2.62 & 40.4 & 465000 & 1.9 & 3.66 \\
\hline Suprofen & 65.8 & 49000 & 18.4 & 1.63 & 47.7 & 134000 & 6.7 & 1.51 & 41.1 & 482000 & 1.9 & 1.46 \\
\hline
\end{tabular}

${ }^{a}$ Column: histidine bonded phase capillary, $90 \mathrm{~cm}(70 \mathrm{~cm}$ to detector $) \times 75 \mu \mathrm{m}$ I.D.; background electrolyte: acetate buffer $(20 \mathrm{mM}, \mathrm{pH}$ 5.0)-organic solvent; injection: hydrodynamic (10 cm, $10 \mathrm{~s})$; sample concentration: $0.5 \mathrm{~m} M$ each, except naproxen $0.25 \mathrm{~m} M$; applied voltage: $-20 \mathrm{kV}$; detection: $\mathrm{UV}$ at $220 \mathrm{~nm}$.

${ }^{\mathrm{b}} N$, Number of theoretical plates $=16\left(t_{\mathrm{R}} / W\right)^{2}$.

${ }^{\mathrm{c}} H$, Height equivalent to theoretical plate.

${ }^{\mathrm{d}} R_{\mathrm{s}}=2\left(t_{\mathrm{R} 2}-t_{\mathrm{R} 1}\right) /\left(W_{1}+W_{2}\right)$.

course, anion exchange would be the predominant interaction force, since all analytes carry carboxylate groups and protonated amino groups are on the bonded phase. As the counter ion for the anion

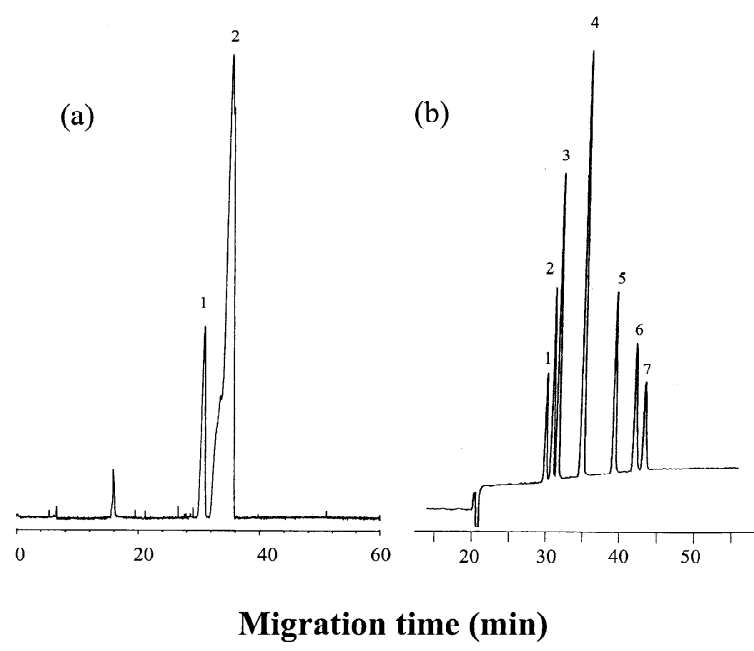

Fig. 6. Comparison the histidine bonded phase with the uncoated capillary column in the separation of NSAIDs. Column: $90 \mathrm{~cm}$ (70 $\mathrm{cm}$ to detector) $\times 75 \mu \mathrm{m}$ I.D., (a) uncoated capillary, (b) histidine bonded phase capillary; background electrolyte: acetate buffer (20 $\mathrm{m} M, \mathrm{pH}$ 5.0)-ethanol (80:20, v/v); sample concentration: $0.5 \mathrm{~m} M$ each, except naproxen $0.25 \mathrm{~m} M$; applied voltage: (a) $+20 \mathrm{kV}$, (b) $-20 \mathrm{kV}$; detection: UV at $220 \mathrm{~nm}$. Peak identification: (a) $1=$ naproxen and flurbiprofen, $2=$ indoprofen, ketoprofen, ibuprofen, fenoprofen and suprofen; (b) $1=$ indoprofen, $2=$ ketoprofen, $3=$ naproxen, $4=$ ibuprofen, $5=$ fenoprofen, $6=$ flurbiprofen, $7=$ suprofen. exchange, indoprofen exhibited the greatest steric hindrance among the analytes. This suggests that it was the analyte with the least affinity for the bonded phase. The increasing order for the hydrogen bonding would be ketoprofen (carbonyl group besides the benzene ring) $<$ naproxen (methyl ether) $<$ fenoprofen (phenyl ether) $<$ flurbiprofen $(\mathrm{F})<$ suprofen $(\mathrm{S}, \mathrm{O})$. The expected order is consistent with the experimental result. Ibuprofen contains an isobutyl group and no high electronegativity atom, this would exhibit only hydrophobicity interaction with the bonded group.

\subsection{Column performance studies}

Due to the strong adsorption between analytes and the bonded phase, an appropriate treatment of the capillary wall prior to the next run is critical to ensure a consistent and repeatable EOF. By changing the washing solvents, it was found that the capillary could be flushed with methanol, pure water and BGE sequentially. The stability of the column expressed by the relative standard deviation (RSD) of migration time for five consecutive injections was less than $1.7 \%$. Given the simplicity of the coating procedures, good reproducibility for the separation was also obtained with an average RSD of migration times for the five different capillaries being $3.7 \%$ as shown in Table 3.

In order to evaluate the potential of the present method for quantitative uses, the linearity of the 
Table 3

Calibration graphs for the determination of NSAIDs ${ }^{a}$

\begin{tabular}{|c|c|c|c|c|c|}
\hline \multirow[t]{2}{*}{ Analyte } & \multirow{2}{*}{$\begin{array}{l}\mathrm{RSD}^{\mathrm{b}} \\
(\%)\end{array}$} & \multicolumn{3}{|c|}{ Linear equation $(y=a x+b)^{\mathrm{c}}$} & \multirow{2}{*}{$\begin{array}{l}\operatorname{LOD}^{\mathrm{d}} \\
\left(\mu M ; \mu \mathrm{g} \mathrm{ml}^{-1}\right)\end{array}$} \\
\hline & & Slope & Intercept & $r^{2}$ & \\
\hline Indoprofen & $1.7^{\mathrm{e}}(3.2)^{\mathrm{f}}$ & 148.78 & 37.29 & 0.9918 & $1.3 ; 0.20$ \\
\hline Ketoprofen & $1.7(3.4)$ & 62.85 & 54.29 & 0.9910 & $1.2 ; 0.25$ \\
\hline Naproxen & $0.9(4.2)$ & 580.07 & 92.66 & 0.9935 & $0.5 ; 0.11$ \\
\hline Ibuprofen & $1.2(4.2)$ & 371.43 & 156.56 & 0.9912 & $0.7 ; 0.14$ \\
\hline Fenoprofen & $1.3(3.4)$ & 668.60 & 38.76 & 0.9958 & $1.2 ; 0.52$ \\
\hline Flurbiprofen & $1.4(3.8)$ & 400.95 & 42.37 & 0.9933 & $1.1 ; 0.24$ \\
\hline Suprofen & $1.7(4.0)$ & 107.07 & 40.20 & 0.9915 & $1.2 ; 0.26$ \\
\hline
\end{tabular}

${ }^{a}$ Column: histidine bonded phase capillary, $90 \mathrm{~cm}(70 \mathrm{~cm}$ to detector) $\times 75 \mu \mathrm{m} \mathrm{I.D.;} \mathrm{background} \mathrm{electrolyte:} \mathrm{acetate} \mathrm{buffer} \mathrm{(pH} 5.0,20$ $\mathrm{mM}$ )-ethanol (80:20, v/v); injection: hydrodynamic $(10 \mathrm{~s}, 10 \mathrm{~cm})$; applied voltage: $-20 \mathrm{kV}$; detection: UV at $220 \mathrm{~nm}$.

${ }^{\mathrm{b}}$ Sample concentration: $0.5 \mathrm{~m} M$ each, except naproxen $0.25 \mathrm{~m} M$.

${ }^{c} y$ : Peak area $(\mu \mathrm{V}$ s), $x$ : concentration $(\mu M)$, linear range: $10 \sim 70 \mu M$.

${ }^{\mathrm{d}} \mathrm{S} / N=3$.

${ }^{\mathrm{e}}$ Run-to-run, $n=5$.

${ }^{\mathrm{f}}$ Column-to-column, $n=5$.

calibration lines was estimated by correlating the peak area $(\mu \mathrm{V}$ s) to sample concentration $(\mu M)$. The results are shown in Table 3. A good linearity was exhibited within the range tested. The detection limits defined as three times signal-to-noise ratio are below $1.3 \mu M$.

\section{Conclusions}

In a preliminary study, histidine bonded phase was much superior to the unsubstituted imidazole bonded phase for the CEC separation of NSAIDs. It was also found that a lower signal and stronger adsorption for the analyte toward the histidine bonded phase by using phosphate buffer-methanol as the BGE. As acetate buffer-methanol was used instead, a more satisfactory result was obtained.

There are several ways for the analyte to interact with the bonded phase. Among them, hydrogen bonding, hydrophobic force and electrostatic force (anion exchange) between analytes and the stationary phase as well as electrophoretic mobility of the analytes all play important roles. Although strong adsorption between analytes and the bonded phase, a satisfactory and reproducible separation could be obtained by a washing procedure with methanol, pure water and BGE between runs.

\section{Acknowledgements}

The authors are thankful to the National Science Council of Taiwan for financial support.

\section{References}

[1] T. Hirai, S. Matsumoto, I. Kishi, J. Chromatogr. B 692 (1997) 375.

[2] M.E. Abdel-Hamid, L. Novotny, H. Hamza, J. Pharm. Biomed. Anal. 24 (2001) 587.

[3] J.R. Veraart, M.C.E. Groot, C. Gooijer, H. Lingeman, N.H. Velthorst, U.A.Th. Brinkman, Analyst 124 (1999) 115.

[4] M.J. Martin, F. Pablos, A.G. Gonzalez, Talanta 49 (1999) 453.

[5] A. Bakkali, E. Corta, L.A. Berrueta, B. Gallo, F. Vicente, J. Chromatogr. B 729 (1999) 139.

[6] A.K. Singh, Y. Jang, U. Mishra, K. Granley, J. Chromatogr. 568 (1991) 351.

[7] A.G. Kazemifard, D.E. Moore, J. Chromatogr. 533 (1990) 125.

[8] S. Toasaksiri, D.L. Massart, Y.V. Heyden, Anal. Chim. Acta 416 (2000) 29.

[9] M. Fillet, I. Bechet, V. Piette, J. Crommen, Electrophoresis 20 (1999) 1907.

[10] M. Blanco, J. Coello, H. Iturriaga, S. Maspoch, C. PerezMaseda, J. Chromatogr. A 793 (1998) 165.

[11] S. Cherkaoui, J.L. Veuthey, J. Chromatogr. A 874 (2000) 121.

[12] C.W. Maboundou, G. Paintaud, M. Berard, P.R. Bechtel, J. Chromatogr. B 657 (1994) 173. 
[13] R. Weinberger, M. Albin, J. Liq. Chromatogr. 14 (1991) 953. [14] A.E. Karger, E. Stoll, W. Hansel, Pharmazie 49 (1994) 155.

[15] C. Mardones, A. Rios, M. Valcarcel, Electrophoresis 22 (2001) 484.

[16] F. Wang, M.G. Khaledi, J. Chromatogr. A 817 (1998) 121.

[17] M. Fillet, P. Hubert, J. Crommen, Electrophoresis 18 (1997) 1013.

[18] X. Zu, B. Lin, U. Epperlein, B. Koppenhoefer, Chirality 11 (1999) 56.
[19] C. Desiderio, S. Fanali, J. Chromatogr. A 895 (2000) 123.

[20] D. Hoegger, R. Freitag, J. Chromatogr. A 914 (2001) 211.

[21] Z. Liu, K. Otsuka, S. Terabe, J. Chromatogr. A 959 (2002) 241.

[22] T.S. Chen, C.Y. Liu, Electrophoresis 22 (2001) 2606.

[23] H.H. Chiang, C.C. Shiue, Y.F. Pai, C.Y. Liu, Instrum. Sci. Technol. 30 (2002) 43.

[24] A. Jouyban-Gharamaleki, M.G. Khaledi, B.J. Clark, J. Chromatogr. A 868 (2000) 277. 